\title{
Optimal Impulse Control for Cash Management with Double Exponential Jump Diffusion Processes*
}

\author{
Kimitoshi Sato ${ }^{\mathrm{a}, \dagger} \quad$ Atsuo Suzuki ${ }^{\mathrm{b}}$ \\ ${ }^{a}$ Faculty of Engineering, Kanagawa University \\ ${ }^{\mathrm{b}}$ Faculty of Urban Science, Meijo University
}

\begin{abstract}
We consider a cash management problem where the cash demand is assumed to be double exponential jump-diffusion processes. We formulate a model minimizing the sum of the transaction and holding-penalty costs as an impulse control model. The model reduces to the problem of solving a Quasi-variational Inequality (QVI), and the function satisfying QVI is derived. We show that there is an optimal policy of the two-band type. Moreover, we discuss the effect of jumps on the optimal policy through some numerical examples.
\end{abstract}

Keywords: Inventory; Cash management; Jump diffusion; Impulse control

\footnotetext{
${ }^{*}$ Received: January 10, 2018; Accepted: November 20, 2018.

${ }^{\dagger}$ Corresponding author. Address: 3-27-1 Rokkakubashi, Kanagawa-ku, Yokohama, Kanagawa, 221-8686, Japan; Phone: +81-45-481-5661; E-mail: k-sato@kanagawa-u.ac.jp
} 


\section{Introduction}

Managing cash is one of the most important issues of a firm. Even if a firm is growing and has good performance, it cannot survive if it runs out of cash. In this paper, we study a cash management model (CMM) faced by a firm which want to manage their holding cash under the possibility of its sudden change. There are two primary reasons for the sudden change in holding cash. One is a large amount of positive demand (disbursements) due to natural or human-caused disaster (i.e. earthquake, tsunami, terrorism and financial crash). The other is a large amount of negative demand (collections) which due to gaining a high profit margin or high return on invested capital. Thus, the firm needs to maintain appropriate cash level as a buffer. In order to investigate the effect of such risks on the management, we formulate a continuous-time model with jump diffusion process, and find an optimal strategy so as to minimize total cost consisting of transaction and holding-penalty costs for the cash.

The CMM has been studied as an extension of the inventory model (Baumol [4], Miller and Orr [13], Constantinides and Richard [8] and Baccarin [1], [2]). Baumol [4] was the first to provide a CMM as an extension of the economic order quantity (EOQ) model incorporating opportunity costs and trading costs. Miller and Orr [13] developed a CMM to deal with cash inflows and outflows that fluctuate randomly from day to day. They assumed that the distribution of daily net cash flow is normally distributed and derived an optimal policy which consists of upper and lower control limits and a target cash balance. Constantinides and Richard [8] and Baccarin [1], [2] formulated a CMM as an impulse control problem in which the demand for cash is generated by a Brownian motion with drift. They derived a value function as a solution of a quasi-variational inequality (QVI) and showed a band-type optimal policy $(d, D, U, u)$. That is, the cash level is adjusted up to level $D$ (down to $U$, resp.), when it falls to $d$ (rise to $u$, resp.), $d<D<U<u$. The existence of an optimal policy were derived through a verification theorem approach by assuming a priori the smooth-fit property through the action / continuation regions. On the other hand, Guo and $\mathrm{Wu}$ [10] proved the smooth-fit $C^{1}$ property of the value function for muti-dimensional controlled diffusions by using a viscosity solution approach. Then, they provided an alternative derivation of optimal policy and value function for the CMM by exploiting the regularity property.

Recently, some works discuss the inventory model used by a jump diffusion process to incorporate a large amount of cash demands. Ber-Ilan et al. [3] used a compound Poisson process to incorporate such demands. However, the existence of policy parameters was not shown in this paper. Bensoussan et al. [7] showed that an $(s, S)$ policy is optimal when the demand is a mixture of a diffusion process and a compound Poisson process with exponentially distributed jumps size. Benkherouf and Bensoussan [5] extended Bensoussan et al. [7] to the general case where the demand is a combination of a diffusion and a general compound Poisson process with nonnegative jump size. In this paper, we also deal with jump diffusion process to represent the large amount of demands. Unlike previous works, we consider not only the process with positive jump size but also that with negative one by assuming the double-exponentially distributed jumps. Then, we derive an explicit formula for the solution of QVI and show that the band policy $(d, D, U, u)$ is optimal using the regularity property.

The rest of this paper is organized as follows: In the next section, we construct a mathematical model to describe the cash management problem. In section 3, we provide a precise formulation of the impulse control model with jump diffusion and refer the result of the viscosity solution and regularity 
property under consideration. In section 4, we solve the QVI to obtain a closed-form expression for the value function and show the existence of the optimal policy. In section 5 , we present numerical studies and the final section concludes the research.

\section{Problem Formulation}

In this section, we consider a cash management model in which the manager who faces with large fluctuation of the cash level is allowed to adjust it to the appropriate level so as to minimize the sum of transaction and holding-penalty costs. Let $(\Omega, \mathcal{F}, P)$ be the underlying probability space. Let $Z_{t}$ be the cumulative demand in the interval $[0, t]$ and is given by

$$
Z_{t}=\mu t+\sigma W_{t}+M_{t},
$$

where $\mu$ is drift, $\sigma$ is the volatility, $W_{t}$ is a standard Brownian motion with $W_{0}=0$ and $M_{t}$ is a compound Poisson process with $M_{0}=0$. The process $M_{t}$ is defined as follows;

$$
M_{t}=\sum_{i=1}^{N_{t}} Y_{i}
$$

We denote that $N_{t}$ is a Poisson process with rate $\lambda \geq 0$, and $Y_{i}, i=1,2, \cdots$, is an i.i.d. sequence of random variables with distribution density $m(\cdot)$. We assume that $W_{t}, N_{t}$ and $Y_{i}$ are all independent. According to the model (1), the cumulate demand consists of three factors: a deterministic trend of demand $\mu$, inherent risk described by the Brownian motion, and exogeneous risk captured by the Poisson-arrival jump part. In this paper, we deal with not only the downward jump but also upward jump. We assume that $Y_{i}$ has a double exponential distribution and its density function is given by

$$
m(y)=p_{u} \eta_{1} e^{-\eta_{1} y} 1_{\{y \geq 0\}}+p_{d} \eta_{2} e^{\eta_{2} y} 1_{\{y<0\}},
$$

where $\eta_{1}, \eta_{2}>0$ and $p_{u}, p_{d} \geq 0$ such that $p_{u}+p_{d}=1$. The mean of $Y_{1}$ is obtained by $\mu_{m} \equiv E\left[Y_{1}\right]=$ $p_{u} / \eta_{1}-p_{d} / \eta_{2}$.

Let $\mathcal{F}_{t}, t>0$, be the sigma algebra generated by $\left\{W_{s}, M_{s}, 0<s \leq t\right\}$ and $\mathcal{F}_{0}=\{\emptyset, \Omega\}$. An admissible impulse control $v$ consists of a sequence of stopping times $\tau_{1}, \tau_{2}, \cdots$ with respect to $\mathcal{F}_{t}$ and a corresponding sequence of $\mathbb{R}$-valued random variables $\xi_{1}, \xi_{2}, \cdots$ satisfying the conditions

$$
\left\{\begin{array}{l}
0<\tau_{1}<\tau_{2}<\cdots<\tau_{i}<\cdots, \\
\tau_{i} \rightarrow \infty, \text { a.s. as } i \rightarrow \infty \\
\xi_{i} \in \mathcal{F}_{\tau_{i}}, \forall i \geq 1
\end{array}\right.
$$

Here, $\tau_{i}$ represents the $i$ th time of adjusting and $\xi_{i}$ represents the quantity adjusted at time $\tau_{i}$. Let $\mathscr{V}$ be the set of admissible impulse control strategies. If the policy $v=\left\{\left(\tau_{i}, \xi_{i}\right), i=1,2, \cdots\right\}$ is adopted, then the cash level at time $t$ evolves as

$$
X_{t}^{v}=x-Z_{t}+\sum_{i=1}^{\infty} I_{\left\{\tau_{i}<t\right\}} \xi_{i}
$$

where $X_{0}^{v}=x$ is an initial cash level at time $t=0$.

When the cash level changes from $x$ to $x+\xi$, the transaction costs occur. We denote a fixed cost by $K_{1}\left(K_{2}\right.$, resp.) and proportional cost by $k_{1}\left(k_{2}\right.$, resp.) if the manager increases (decreases, resp.) 
the cash. Transaction costs function with the cash adjustment $\xi_{i}$ is denoted by the sum of fixed and proportional costs of the form

$$
T\left(\xi_{i}\right)= \begin{cases}K_{1}+k_{1} \xi_{i}, & \text { if } \xi_{i} \geq 0 \\ K_{2}-k_{2} \xi_{i}, & \text { if } \xi_{i}<0\end{cases}
$$

where $K_{1}, K_{2}, k_{1}, k_{2}>0$. Holding and penalty costs function $C(x)$ is defined by

$$
C(x)= \begin{cases}h x, & \text { if } x \geq 0 \\ -p x, & \text { if } x \leq 0\end{cases}
$$

where $x$ is the cash level and $h, p>0$.

Given initial cash level $x$ and control $v$, the associated total expected cost over an infinite planning horizon can be described as

$$
J_{x}(v)=E_{x}^{v}\left[\int_{0}^{\infty} C\left(X_{t}^{v}\right) e^{-\alpha t} d t+\sum_{i=1}^{\infty} T\left(\xi_{i}\right) e^{-\alpha \tau_{i}} \mid X_{0}^{v}=x\right],
$$

where $\alpha>0$ is discount rate. Our objective is to find the policy $v^{*}$ to minimize the expected total cost with the value function

$$
\phi(x)=\inf _{v \in \mathscr{V}} J_{x}(v)
$$

By dynamic programming principle, the value function is associated with the Quasi-Variational Inequality (QVI) which is given by

$$
\max \{\mathcal{L} \phi(x)-C(x)-\mathcal{K} \phi(x), \phi(x)-\mathcal{M} \phi(x)\}=0,
$$

where

$$
\begin{aligned}
(\mathcal{M} \phi)(x) & =\inf _{\xi}\{T(\xi)+\phi(x+\xi)\} \\
(\mathcal{L} \phi)(x) & =-\frac{1}{2} \sigma^{2} \phi^{\prime \prime}(x)+\mu \phi^{\prime}(x)+\alpha \phi(x) \\
(\mathcal{K} \phi)(x) & =\lambda \int_{-\infty}^{\infty}(\phi(x-y)-\phi(x)) m(y) d y .
\end{aligned}
$$

Appendix A.1 provides the derivation of (10).

\section{The Impulse Control of Jump Diffusions}

The quasi-variational inequality, or HJB, (10) is studied by Øksendal and Sulem [14]. They show the value function defined in (9) is a solution to the QVI in a viscosity sense under some conditions, and also show regularity of the value function. In this section we provide related results from the general theory of QVI for impulse control problem with an one-dimensional jump diffusion process. We will show an optimal policy for the cash management problem in Section 4 by using the results.

Let $X(t)$ be the stochastic process when an admissible control policy $v=\left(\tau_{1}, \xi_{1} ; \tau_{2}, \xi_{2} ; \cdots\right)$ is adopted. Then, the process evolves as

$$
d X(t)=\mu\left(X\left(t^{-}\right)\right) d t+\sigma\left(X\left(t^{-}\right)\right) d W(t)+\int_{\mathbb{R}} j\left(X\left(t^{-}\right), z\right) \tilde{N}(d t, d z)+\sum_{i} \delta\left(t-\tau_{i}\right) \xi_{i} .
$$


Here $N(\cdot, \cdot)$ denotes a Poisson random measure. The Lévy measure $m(\cdot):=E(N(1, \cdot))$ may be unbounded and $\tilde{N}(d t, d z)$ is its compensated Poisson random measure with $\tilde{N}(d t, d z):=N(d t, d z)-$ $m(d z) d t$. The function $\delta(\cdot)$ is the Dirac delta function. With this given control, the associated total expected cost $J_{x}(v)$ and value function $\phi(x)$ are defined as the same form of (8) and (9), respectively. Note that the cost functions $C$ and $T$ satisfies the following assumptions.

Assumption 1 (i) Lipschitz conditions on $\mu, \sigma, j$ : there exist constants $I_{\mu}, I_{\sigma}>0$ and a positive function $I_{j}(\cdot) \in L^{1} \cap L^{2}(\mathbb{R}, m)$ such that

$$
\left\{\begin{array}{l}
|\mu(x)-\mu(y)| \leq I_{\mu}|x-y|, \\
|\sigma(x)-\sigma(y)| \leq I_{\sigma}|x-y|, \\
|j(x, z)-j(y, z)| \leq I_{j}(z)|z-y|
\end{array}\right.
$$

Assume also that

$$
j(x, \cdot) \in L^{1}(\mathbb{R}, m), \quad \text { for every } x \in \mathbb{R} .
$$

(ii) Lipschitz condition on the running cost $C \geq 0$ : there exists a constant $I_{C}>0$ such that

$$
|C(x)-C(y)| \geq I_{C}|x-y| .
$$

(iii) Conditions on the transaction cost function $T$ :

$$
\left\{\begin{array}{l}
\inf _{\xi \in \mathbb{R}} T(\xi)=K>0, \\
T \in C(\mathbb{R} \backslash\{0\}), \\
|T(\xi)| \rightarrow \infty, \text { as }|\xi| \rightarrow \infty, \\
T\left(\xi_{1}\right)+T\left(\xi_{2}\right) \geq T\left(\xi_{1}+\xi_{2}\right)+K .
\end{array}\right.
$$

(iv) $\alpha>2 I_{\mu}+I_{\sigma}^{2}+\int_{\mathbb{R}} I_{j}^{2}(z) m(d z)$

Let $\boldsymbol{B}_{0}$ be the family of Borel sets $\mathcal{U} \in \mathbb{R}$ whose closure $\overline{\mathcal{U}}$ does not contain 0 . We adopt the following standard notations for function spaces.

- $U C(\mathbb{R})=$ space of all uniformly continuous functions on $\mathbb{R}$,

- $W^{k, p}(\mathcal{U})=$ space of all $L^{p}$ functions with $\beta$-th weak partial derivatives belonging to $L^{p}, \forall|\beta| \leq k$, where $\mathcal{U} \in \boldsymbol{B}_{0}$,

- $W_{l o c}^{k, p}(\mathcal{U})=\left\{f \in W^{k, p}\left(\mathcal{U}^{\prime}\right), \forall \operatorname{compact} \mathcal{U}^{\prime} \subset \mathcal{U}\right\}$,

- $C^{k, \alpha}(D)=\left\{f \in C^{k}(D): \sup _{x, y \in D, x \neq y}\left\{\frac{\left|D^{\beta} f(x)-D^{\beta} f(y)\right|}{|x-y|^{\alpha}}\right\}<\infty, \forall|\beta| \leq k\right\}, D$ compact.

Definition 1 A function $\phi(\cdot) \in U C(\mathbb{R})$ is called a viscosity subsolution (supersolution, resp.) of QVI if whenever $\psi \in C^{2}(\mathbb{R}), \phi-\psi$ has a local maximum (minimum, resp.) at $x_{0}$ and $\phi\left(x_{0}\right)=\psi\left(x_{0}\right)$, we have

$$
\max \left\{\mathcal{L} \psi\left(x_{0}\right)-C\left(x_{0}\right)-\mathcal{K} \phi\left(x_{0}\right), \phi\left(x_{0}\right)-\mathcal{M} \phi\left(x_{0}\right)\right\} \leq 0(\geq 0, \text { resp. }) .
$$

$\phi$ is called a viscosity solution of QVI if it is both a subsolution and a supersolution. 
Theorem 1 (Øksendal and Sulem [14]) The value function $\phi(\cdot)$ defined by (9) is a viscosity solution of $Q V I(10)$.

Under Assumption 1 (i)-(iv), the following results are shown by Davis et al. [9].

Lemma 1 The value function $\phi(\cdot)$ is Lipschitz.

Lemma 2 Suppose $m(\mathbb{R})<\infty$, then the operator $\mathcal{K}$ maps a Lipschitz function to a Lipschitz function, that is,

$$
|\mathcal{K} \phi(x)-\mathcal{K} \phi(y)| \leq I_{\phi}|x-y|, \text { for any } x, y,
$$

where $I_{\phi}$ is a constant.

Theorem 2 ( $W_{\text {loc }}^{2, p}$-Regularity, Davis et al. [9]) Assume that $m(\mathbb{R})<\infty$ and $\sigma \in C^{1,1}(D)$ for any compact set $D \subset \mathbb{R}$. Then for any bounded open set $\mathcal{O} \subset \mathbb{R}$ and $p<\infty$, we have $\phi \in W^{2, p}(\mathcal{O})$. In particular, $\phi \in C^{1}(\mathbb{R})$ by Sobolev embedding.

The above theorem implies that the value function satisfies a smooth-fit property through the boundaries between action and continuation regions.

\section{Optimal Policy of Cash Management Problem}

In this section we show an optimal policy for the problem described in Section 2. Since our setting of the process $X_{t}^{v}$ in (5) and cost functions $T(\cdot)$ and $C(\cdot)$ satisfy Assumption 1, we can exploit the regularity property in Theorem 2. (See Appendix A.2 for confirmation).

Below is the assumption to show the optimal policy.

Assumption 2 The holding and penalty costs satisfy the following conditions:

(i) $h-\alpha k_{2}>0$;

(ii) $p-\alpha k_{1}>0$.

The Assumption (i) can be rewritten as $h / \alpha>k_{2}$. It implies that the present value of the holding cost of keeping one unit of cash from now to infinity $h / \alpha$ is larger than the proportional cost $k_{2}$. If the relation does not hold, it will never be optimal to adjust the cash level. Similarly, the assumption (ii) is needed to increase the cash level.

Theorem 3 Suppose that Assumption 2 holds.

(i) There exist constants $-\infty<d<u<\infty$ such that

$$
\begin{aligned}
& \mathcal{C}:=\{x \in \mathbb{R}: \phi(x)<\mathcal{M} \phi(x)\}=(d, u) \\
& \mathcal{A}:=\{x \in \mathbb{R}: \phi(x)=\mathcal{M} \phi(x)\}=(-\infty, d] \cup[u,+\infty)
\end{aligned}
$$


(ii) The value function $\phi(x)$ satisfies

$$
\begin{cases}\mathcal{L} \phi(x)-\mathcal{K} \phi(x)=C(x), & \text { if } d<x<u, \\ \phi(x)=\phi(d)+k_{1}(d-x), & \text { if } x \leq d, \\ \phi(x)=\phi(u)+k_{2}(x-u), & \text { if } x \geq u,\end{cases}
$$

(iii) There is a value $D, U \in(d, u)$ such that

$$
\begin{aligned}
\phi^{\prime}(d) & =\phi^{\prime}(D)=-k_{1}, \\
\phi^{\prime}(u) & =\phi^{\prime}(U)=k_{2}, \\
\phi(d) & =\phi(D)+K_{1}+k_{1}(D-d), \\
\phi(u) & =\phi(U)+K_{2}-k_{2}(U-u),
\end{aligned}
$$

We divide the proof of Theorem 3 into a sequence of lemmas, and these steps follow Section 5 of Guo and $\mathrm{Wu}[10]$. The following lemmas represent the properties of the continuation region $\mathcal{C}$ and the action region $\mathcal{A}$, and these are proved in Proposition 1 and Proposition 2 of Guo and $\mathrm{Wu}[10]$.

Lemma $3 \mathcal{C}$ is open.

Lemma 4 Suppose $x \in \mathcal{A}$, then

(1) The set

$$
\Xi(x):=\{\xi \in \mathbb{R}: \mathcal{M} \phi(x)=\phi(x+\xi)+T(\xi)\}
$$

is nonempty, i.e., the infimum is in fact a minimum.

(2) Moreover, for any $\xi(x) \in \Xi(x)$, we have

$$
\phi(x+\xi(x)) \leq \mathcal{M} \phi(x+\xi(x))-K,
$$

in particular,

$$
x+\xi(x) \in \mathcal{C} .
$$

This lemma guarantee the existence of the positive quantity to minimize the total expected cost at the time of cash adjustment, $\xi(x)>0$ for $x \in \mathcal{A}$. The next lemmas provide the slope of value function $\phi(\cdot)$ before and after the cash adjustment. Guo and $\mathrm{Wu}[10]$ show the same properties where the underlying process is a diffusion process without jumps. The same results hold for the process with jumps. Since the proofs are quite similar to Lemmas 5.2 and 5.3 of Guo and $\mathrm{Wu}$ [10], we omit the proofs here.

Lemma 5 (Lemma 5.2 of Guo and $\mathbf{W u}[\mathbf{1 0}]$ ) For any $x_{0} \in \mathcal{A}$ and $\xi_{0} \in \Xi\left(x_{0}\right)$, we have

$$
\phi^{\prime}\left(x_{0}\right)=\phi^{\prime}\left(x_{0}+\xi_{0}\right)= \begin{cases}-k_{1}, & \text { if } \xi_{0} \geq 0 \\ k_{2}, & \text { if } \xi_{0}<0 .\end{cases}
$$


From this lemma, we see that the slope is negative (positive) if the cash level is increased (decreased) by the adjustment.

Lemma 6 (Lemma 5.3 of Guo and Wu [10]) For any $x_{0} \in \mathcal{A}$ and $\xi_{0} \in \Xi\left(x_{0}\right)$,

(i) if $x_{0}>0$, then $\xi_{0}<0$ and $\phi^{\prime}\left(x_{0}\right)=k_{2}$;

(ii) if $x_{0}<0$, then $\xi_{0}>0$ and $\phi^{\prime}\left(x_{0}\right)=-k_{1}$.

This lemma provides the relationship between the cash level and the quantity of cash adjustment. If the cash level is large enough (scarce), then the cash level is decreased (increased) due to limit the cost increases.

Lemma 7 For any $x \in \mathcal{C}$, the integro-differential equation $\mathcal{L} \phi-\mathcal{K} \phi=C$ has a general solution

$$
\phi(x)= \begin{cases}A_{1} e^{\beta_{1} x}+A_{2} e^{\beta_{2} x}-\frac{p}{\alpha} x+\frac{p}{\alpha^{2}}\left(\mu+\lambda \mu_{m}\right), & \text { if } x \leq 0, \\ A_{3} e^{\beta_{3} x}+A_{4} e^{\beta_{4} x}+\frac{h}{\alpha} x-\frac{h}{\alpha^{2}}\left(\mu+\lambda \mu_{m}\right), & \text { if } 0 \leq x .\end{cases}
$$

Values $\beta_{i}, i=1,2,3,4$, are solution of the equation $G(\theta)=\alpha$ where

$$
G(\theta)=-\mu \theta+\frac{1}{2} \sigma^{2} \theta^{2}+\lambda\left(\frac{p_{u} \eta_{1}}{\eta_{1}+\theta}+\frac{p_{d} \eta_{2}}{\eta_{2}-\theta}-1\right)
$$

and

$$
-\infty<\beta_{4}<-\eta_{1}<\beta_{3}<0<\beta_{2}<\eta_{2}<\beta_{1}<\infty
$$

The constants $A_{j}, j=1, \cdots, 4$, are solutions of the equations

$$
\left(\begin{array}{cccc}
1 & 1 & -1 & -1 \\
\beta_{1} & \beta_{2} & -\beta_{3} & -\beta_{4} \\
\frac{1}{\eta_{1}+\beta_{1}} & \frac{1}{\eta_{1}+\beta_{2}} & -\frac{1}{\eta_{1}+\beta_{3}} & -\frac{1}{\eta_{1}+\beta_{4}} \\
\frac{1}{\eta_{2}-\beta_{1}} & \frac{1}{\eta_{2}-\beta_{2}} & -\frac{1}{\eta_{2}-\beta_{3}} & -\frac{1}{\eta_{2}-\beta_{4}}
\end{array}\right)\left(\begin{array}{c}
A_{1} \\
A_{2} \\
A_{3} \\
A_{4}
\end{array}\right)=\left(\begin{array}{c}
-\frac{1}{\alpha^{2}}(h+p)\left(\mu+\lambda \mu_{m}\right), \\
\frac{1}{\alpha}(h+p), \\
-\frac{1}{\eta_{1}^{2} \alpha^{2}}(h+p)\left(\alpha+\eta_{1}\left(\mu+\lambda \mu_{m}\right)\right), \\
\frac{1}{\eta_{2}^{2} \alpha^{2}}(h+p)\left(\alpha-\eta_{2}\left(\mu+\lambda \mu_{m}\right)\right) .
\end{array}\right)
$$

Proof: First, we solve the homogeneous ordinary differential equation (ODE), $\mathcal{L} \phi-\mathcal{K} \phi=0$. We guess that the solution has a form $\hat{\phi}(x)=e^{\theta x}$. Plugging it into $\mathcal{L} \phi-\mathcal{K} \phi=0$, we get the corresponding characteristic equation $G(\theta)=\alpha$ where $G(\cdot)$ is defined by (33). Next, we find a particular solution for non-homogeneous ODE, $\mathcal{L} \phi-\mathcal{K} \phi=C$. For $x \leq 0$, we guess that the solution has a form $\psi_{1}(x)=a_{1} x+b_{1}$. Substituting it into equation $\mathcal{L} \phi-\mathcal{K} \phi=C$, we obtain $a_{1}=-\frac{p}{\alpha}, b_{1}=\frac{p}{\alpha^{2}}\left(\mu+\lambda \mu_{m}\right)$, where $\mu_{m}$ is the expectation of the random variable $Y_{1}$. Thus, the general solution has the form

$$
\phi(x)= \begin{cases}A_{1} e^{\beta_{1} x}+A_{2} e^{\beta_{2} x}+\psi_{1}(x), & \text { if } x \leq 0, \\ A_{3} e^{\beta_{3} x}+A_{4} e^{\beta_{4} x}+\psi_{2}(x), & \text { if } 0 \leq x,\end{cases}
$$

where $\beta_{i}, i=1,2,3,4$, are solution of equation $G(\theta)=\alpha$.

Following Kou and Wang [12], we show that the existence of four roots of an equation $G(\theta)=\alpha$ for any $\alpha>0$. Define

$$
G_{0}(\theta) \equiv-\mu \theta+\frac{1}{2} \sigma^{2} \theta^{2}+\lambda\left(\frac{p_{u} \eta_{1}}{\eta_{1}+\theta}+\frac{p_{d} \eta_{2}}{\eta_{2}-\theta}-1\right)-\alpha
$$


Since we have

$$
G_{0}^{\prime \prime}(\theta)=2 \lambda\left(\frac{p_{u} \eta_{1}}{\left(\eta_{1}+\theta\right)^{3}}+\frac{p_{d} \eta_{2}}{\left(\eta_{2}-\theta\right)^{3}}\right)+\sigma^{2} .
$$

$G_{0}(\theta)$ is a convex function on the interval $\left(-\eta_{1}, \eta_{2}\right)$. From $G_{0}(0)=-\alpha<0, G_{0}(\theta) \rightarrow \infty$ as $\theta \downarrow-\eta_{1}$ and $G_{0}(\theta) \rightarrow \infty$ as $\theta \uparrow \eta_{2}$, there is exactly one root $\beta_{3}$ for $G(\theta)=\alpha$ on the interval $\left(-\eta_{1}, 0\right)$, and the another one $\beta_{2}$ on the interval $\left(0, \eta_{2}\right)$. Similarly, there is at least one on $\left(-\infty,-\eta_{1}\right)$, as $G_{0}(\theta) \rightarrow \infty$ as $\theta \rightarrow-\infty$ and $G_{0}(\theta) \rightarrow-\infty$ as $\theta \uparrow-\eta_{1}$. But the equation $G(\theta)=\alpha$ is actually a polynomial equation with degree four. Therefore, it can have at most four real roots. It follows that there is exactly one root on each interval $\left(-\infty,-\eta_{1}\right)$ and $\left(\eta_{2}, \infty\right)$.

To determine $A_{i}, i=1, \cdots, 4$, we require that $\phi(x)$ and $\phi^{\prime}(x)$ are continuous at $x=0$, that is,

$$
\left\{\begin{array}{l}
\left.\phi(x)\right|_{x=0^{-}}=\left.\phi(x)\right|_{x=0^{+}}, \\
\left.\phi^{\prime}(x)\right|_{x=0^{-}}=\left.\phi^{\prime}(x)\right|_{x=0^{+}} .
\end{array}\right.
$$

It can be rewritten as

$$
\left\{\begin{array}{l}
A_{1}+A_{2}-A_{3}-A_{4}=-\frac{1}{\alpha^{2}}\left(\mu+\lambda \mu_{m}\right)(p+h), \\
A_{1} \beta_{1}+A_{2} \beta_{2}-A_{3} \beta_{3}-A_{4} \beta_{4}=\frac{1}{\alpha}(p+h) .
\end{array}\right.
$$

For $x \leq 0$, we substitute (32) into equation $\mathcal{L} \phi-\mathcal{K} \phi=C$, that is,

$$
-\frac{1}{2} \sigma^{2} \phi^{\prime \prime}(x)+\mu \phi^{\prime}(x)+(\lambda+\alpha) \phi(x)-\lambda \int_{-\infty}^{\infty} \phi(x-y) m(y) d y-C(x)=0
$$

The fourth term of above equation is given by

$$
\begin{aligned}
\int_{-\infty}^{\infty} \phi(x-y) m(y) d y=\int_{-\infty}^{x} \phi(x-y) m(y) & d y \\
& +\int_{x}^{0} \phi(x-y) m(y) d y+\int_{0}^{\infty} \phi(x-y) m(y) d y
\end{aligned}
$$

where

$$
\begin{aligned}
\int_{-\infty}^{x} \phi(x-y) m(y) d y= & p_{d} \eta_{2} e^{\eta_{2} x} \sum_{i=3}^{4} \frac{A_{i}}{\eta_{2}-\beta_{i}}+p_{d} \frac{h}{\alpha} e^{\eta_{2} x}\left\{\frac{1}{\eta_{2}}-\frac{1}{\alpha}\left(\mu+\lambda \mu_{m}\right)\right\} \\
\int_{x}^{0} \phi(x-y) m(y) d y= & p_{d} \eta_{2} \sum_{i=1}^{2} \frac{A_{i}}{\eta_{2}-\beta_{i}}\left(e^{\beta_{i} x}-e^{\eta_{2} x}\right) \\
& -p_{d} \frac{p}{\alpha}\left[x+\left(\frac{1}{\eta_{2}}-\frac{1}{\alpha}\left(\mu+\lambda \mu_{m}\right)\right)\left(1-e^{\eta_{2} x}\right)\right], \\
\int_{0}^{\infty} \phi(x-y) m(y) d y= & p_{u} \eta_{1} \sum_{i=1}^{2} \frac{A_{i}}{\beta_{i}+\eta_{1}} e^{\beta_{i} x}-p_{u} \frac{p}{\alpha}\left\{x-\frac{1}{\eta_{1}}-\frac{p}{\alpha}\left(\mu+\lambda \mu_{m}\right)\right\} .
\end{aligned}
$$

Since $\beta_{1}$ and $\beta_{2}$ are the solutions of $G(\theta)=\alpha$ where $G(\cdot)$ is defined by (33), (41) can be rewritten as

$$
\sum_{i=1}^{2} \frac{A_{i}}{\eta_{2}-\beta_{i}}-\sum_{i=3}^{4} \frac{A_{i}}{\eta_{2}-\beta_{i}}=\frac{1}{\eta_{2}^{2} \alpha^{2}}(h+p)\left(\alpha-\eta_{2}\left(\mu+\lambda \mu_{m}\right)\right) .
$$

In the same manner for $x \geq 0$, we get

$$
\sum_{i=1}^{2} \frac{A_{i}}{\beta_{i}+\eta_{1}}-\sum_{i=3}^{4} \frac{A_{i}}{\beta_{i}+\eta_{1}}=-\frac{1}{\eta_{1}^{2} \alpha^{2}}(h+p)\left(\alpha+\eta_{1}\left(\mu+\lambda \mu_{m}\right)\right) .
$$

Therefore, the system (35) follows from (40), (46) and (47). 
Lemma 8 Under Assumption 2, the continuous region $\mathcal{C}$ does not contain any of the intervals $(-\infty, d)$ or $(u,+\infty)$ with $d \leq+\infty$ and $-\infty \leq u$.

Proof: Suppose $(-\infty, d) \subset \mathcal{C}$. For $c<\min \{d, 0\}$, we have $\mathcal{L} \phi-\mathcal{K} \phi=C$ for $x \in(-\infty, c)$. It follows from Lemma 7 that the general solution is given by

$$
\phi(x)=A_{1} e^{\beta_{1} x}+A_{2} e^{\beta_{2} x}-\frac{p}{\alpha} x+\frac{p}{\alpha^{2}}\left(\mu+\lambda \mu_{m}\right) .
$$

Since there exists a value $\xi \in \Xi(x)$ by Lemma 4 , for any $x<c<0$, we have

$$
\begin{aligned}
\phi(x)<\mathcal{M} \phi(x) & =\inf _{\xi}\{\phi(x+\xi)+T(\xi)\} \\
& \leq \phi(c)+T(c-x)=\phi(c)+K_{1}+k_{1}(c-x),
\end{aligned}
$$

where the second inequality follows from Lemma 4 because there exists a value $\xi \in \Xi(x)$.

The inequality can be rewritten as

$$
A_{1} e^{\beta_{1} x}+A_{2} e^{\beta_{2} x}-\left(\frac{p}{\alpha}-k_{1}\right) x<\phi(c)+K_{1}+k_{1} c-\frac{p}{\alpha^{2}}\left(\mu+\lambda \mu_{m}\right) .
$$

By Assumption 2 (ii), the left-hand side of (50) goes to $+\infty$ as $x \rightarrow-\infty$. It contradicts the fact that $\phi(x)$ is Lipschitz by Lemma 1 . Hence, $\mathcal{C}$ can not contain the interval $(-\infty, d)$.

Similarly, we can show that $\mathcal{C}$ does not contain intervals of $(u,+\infty)$ under Assumption 2 (i). Therefore, the proof is complete.

Lemma 9 The continuous region $\mathcal{C}$ is connected.

Proof: Suppose the assertion of the lemma is false. There are points $y_{1}<y_{2}<y_{3}$ so that $y_{1}, y_{3} \in \mathcal{C}$ while $y_{2} \in \mathcal{A}$. Define

$$
\begin{aligned}
& x_{1}:=\inf \left\{x \in \mathcal{A}: x \leq y_{2},\left[x, y_{2}\right] \subset \mathcal{A}\right\}, \\
& x_{2}:=\sup \left\{x \in \mathcal{A}: x \geq y_{2},\left[y_{2}, x\right] \subset \mathcal{A}\right\} .
\end{aligned}
$$

By Lemma 5, we have $\phi^{\prime}(x)=-k_{1}$ or $k_{2}$ for all $x \in \mathcal{A}$. In addition, from Theorem 2 , we have $\phi \in C^{1}(\mathbb{R})$. Thus, $\phi^{\prime}$ is a constant on $\left[x_{1}, x_{2}\right]$. Here, we assume that $\phi^{\prime}(x)=-k_{1}$ for all $x \in\left[x_{1}, x_{2}\right] \in \mathcal{A}$ and consider $\phi(\cdot)$ at the point $x_{1}$. Let $\xi_{1} \in \Xi\left(x_{1}\right)$ be the amount of adjustment when the cash level $x_{1}$. Then, we have $\xi_{1} \geq 0$ by Lemma 5 . For $x \leq x_{1}$, we obtain

$$
\begin{aligned}
\phi(x) & \leq \mathcal{M} \phi(x) \\
& =\inf _{\xi}\{\phi(x+\xi)+T(\xi)\} \\
& =\inf _{y}\left\{\phi\left(x+\xi_{1}+y\right)+T\left(\xi_{1}+y\right)\right\} \\
& \leq \phi\left(x_{1}+\xi_{1}\right)+T\left(\xi_{1}+x_{1}-x\right) \\
& =\phi\left(x_{1}+\xi_{1}\right)+T\left(\xi_{1}\right)-k_{1}\left(x-x_{1}\right) \\
& =\phi\left(x_{1}\right)-k_{1}\left(x-x_{1}\right),
\end{aligned}
$$

where the second inequality follows from Lemma 4. The inequality is strict if $x<x_{1}$ and $x \in \mathcal{C}$. Similarly, the case of $\phi^{\prime}(x)=k_{2}$ is shown by considering $\phi(x)$ at the point $x_{2}$. 


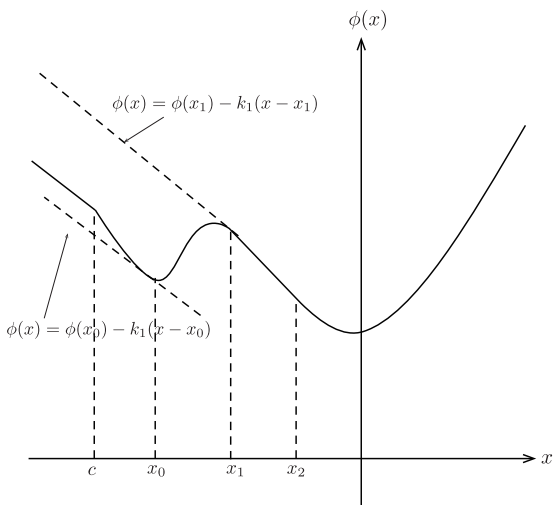

Figure 1: Form of the candidate function $\phi(x)$ in Figure 2: Form of the candidate function $\phi^{\prime}(x)$ the proof of Lemma 9 .

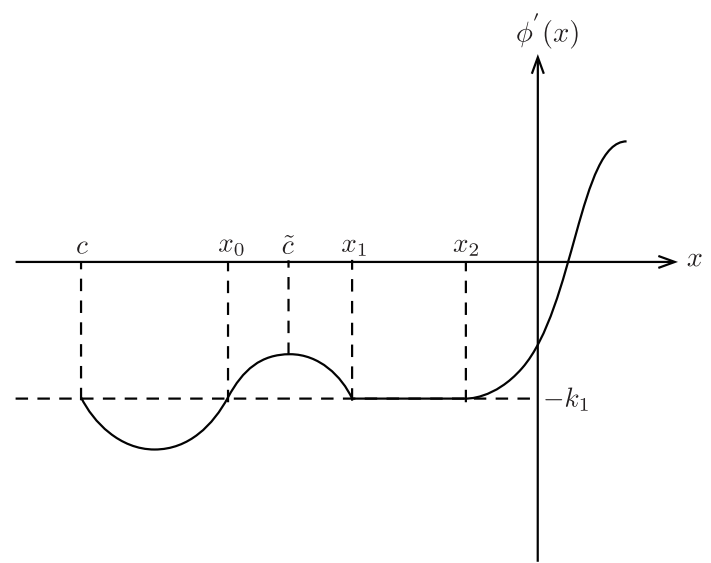

in the proof of Lemma 9.

Define $\Phi(x) \equiv \phi\left(x_{1}\right)-k_{1}\left(x-x_{1}\right)$ for $x \leq x_{1}$ which is uniformly continuous. It follows from the inequality (53) that

$$
\phi\left(x_{1}\right)-\Phi\left(x_{1}\right)-(\phi(x)-\Phi(x))=-\phi(x)+\phi\left(x_{1}\right)-k_{1}\left(x-x_{1}\right) \geq 0 .
$$

Thus, $\phi\left(x_{1}\right)-\Phi\left(x_{1}\right) \geq \phi(x)-\Phi(x)$ for $x \leq x_{1}$, which implies that $x_{1}$ is a local maximum of $\phi-\Phi$. By the viscosity subsolution property which is Definition 1, we have

$$
-\frac{1}{2} \sigma^{2} \Phi^{\prime \prime}\left(x_{1}\right)+\mu \Phi^{\prime}\left(x_{1}\right)+\alpha \Phi\left(x_{1}\right)-\mathcal{K} \phi\left(x_{1}\right)-C\left(x_{1}\right) \leq 0 .
$$

Since $\Phi^{\prime}\left(x_{1}\right)=-k_{1}, \Phi^{\prime \prime}\left(x_{1}\right)=0$ and $\phi\left(x_{1}\right)=\Phi\left(x_{1}\right)$, the inequality (55) can be rewritten as

$$
-\mu k_{1}+\alpha \phi\left(x_{1}\right)-\mathcal{K} \phi\left(x_{1}\right) \leq C\left(x_{1}\right) .
$$

Suppose $\left(c, x_{1}\right)$ is an open interval component of $\mathcal{C}$. Then by Lemma $8, \mathcal{C}$ does not contain the interval $(-\infty, d)$, so $c<\infty$ and thus $(-\infty, c] \subset A$. From Lemma 5 and $c \in A$, we have $\phi^{\prime}(c)=-k_{1}$. Since $\phi(x)<\phi\left(x_{1}\right)-k_{1}\left(x-x_{1}\right)$ for $x<x_{1}$ by (53), there exists $\tilde{c} \in\left(c, x_{1}\right)$ such that $\phi^{\prime}(\tilde{c})>-k_{1}$. Now, we define

$$
x_{0}=\inf \left\{c \leq x \leq \tilde{c}: \phi^{\prime}(x)=-k_{1}\right\} .
$$

Figures 1 and 2 illustrate the form of the function $\phi(x)$ and $\phi^{\prime}(x)$, respectively. Under the assumption of $\left(c, x_{1}\right) \in \mathcal{C}$, we see that there exists a value $x_{0}$ in $c \leq x \leq \tilde{c}$ by Figure 2, since $\phi^{\prime}\left(x_{1}\right)=\phi^{\prime}(c)=-k_{1}$ and $\phi(x)<\phi\left(x_{1}\right)-k_{1}\left(x-x_{1}\right)$ for $x<x_{1}$. The form of function $\phi(x)$ corresponds to Figure 1 . We see that $x_{0} \leq \tilde{c}<x_{1}$ and $\phi^{\prime}(x)>-k_{1}=\phi^{\prime}\left(x_{0}\right)$ for $x_{0}<x \leq \tilde{c}$. Thus, we obtain $\phi^{\prime \prime}\left(x_{0}\right) \geq 0$. Hence, the equation $\mathcal{A} \phi\left(x_{0}\right)=C\left(x_{0}\right)$ is given by

$$
\begin{aligned}
C\left(x_{0}\right) & =-\frac{1}{2} \sigma^{2} \phi^{\prime \prime}\left(x_{0}\right)+\mu \phi^{\prime}\left(x_{0}\right)+\alpha \phi\left(x_{0}\right)-\mathcal{K} \phi\left(x_{0}\right) \\
& \leq-\mu k_{1}+\alpha \phi\left(x_{0}\right)-\mathcal{K} \phi\left(x_{0}\right) .
\end{aligned}
$$

Since $x_{0}, x_{1} \leq 0$, we have $C\left(x_{0}\right)=-p x_{0}$ and $C\left(x_{1}\right)=-p x_{1}$. Thus, it follows from (56) and (58) that

$$
\phi\left(x_{0}\right)-\phi\left(x_{1}\right)>\frac{p}{\alpha}\left(x_{1}-x_{0}\right)+\frac{1}{\alpha}\left\{\mathcal{K} \phi\left(x_{0}\right)-\mathcal{K} \phi\left(x_{1}\right)\right\} .
$$


From the property of the operator $\mathcal{K}$ in Lemma 2, we have

$$
-I_{\phi}\left|x_{0}-x_{1}\right| \leq \mathcal{K} \phi\left(x_{0}\right)-\mathcal{K} \phi\left(x_{1}\right) \leq I_{\phi}\left|x_{0}-x_{1}\right| .
$$

It follows from $x_{0}<x_{1}$ that

$$
\frac{1}{\alpha}\left(\mathcal{K} \phi\left(x_{0}\right)-\mathcal{K} \phi\left(x_{1}\right)\right) \geq-\frac{1}{\alpha} I_{\phi}\left(x_{1}-x_{0}\right) .
$$

Thus, by taking a small value $I_{\phi}$, we obtain

$$
\phi\left(x_{0}\right)-\phi\left(x_{1}\right)>\frac{1}{\alpha}\left(x_{1}-x_{0}\right)\left(p-I_{\phi}\right)>k_{1}\left(x_{1}-x_{0}\right),
$$

where the second inequality follows from Assumption 2 (ii), $p_{1}-\alpha k_{1}>0$. Therefore, it is a contradiction to (53).

Finally, we show Theorem 3.

Proof: $\quad$ (i) Since $\mathcal{C}$ is connected, by Lemma 8, we have $\mathcal{C}=(d, u)$ for a $-\infty<d<u<\infty$.

(ii) For any $x \leq d$, the amount of adjustment is $\xi \in \Xi(x)$. Since the cash level is in the continuous region $x+\xi \in \mathcal{C}=(d, u)$ after the adjustment, we have $\xi>0$. From Lemma 5 , we obtain $\phi^{\prime}(x)=$ $-k_{1}$. Hence, $\phi(x)=\phi(d)+k_{1}(d-x)$ for $x \leq d$. Similarly, we obtain $\phi(x)=\phi(u)+k_{2}(x-u)$ for $u \leq x$.

(iii) Let $\xi \in \Xi(d)$ and $D=d+\xi$. Then, we have $D \in(d, u), \phi^{\prime}(d)=\phi^{\prime}(D)=-k_{1}$ and

$$
\phi(d)=\mathcal{M} \phi(d)=\phi(D)+T(D-d)=\phi(D)+K_{1}+k_{1}(D-d) .
$$

Next, we derive the value function which satisfies (23) with boundary conditions (24), (25), (26) and (27).

Proposition 1 The function $\phi$ satisfying (23) is given by

$$
\phi(x)= \begin{cases}\phi(d)+k_{1}(d-x), & \text { if } x \leq d, \\ \left(A_{1}+A_{5}\right) e^{\beta_{1} x}+\left(A_{2}+A_{6}\right) e^{\beta_{2} x}+A_{7} e^{\beta_{3} x}+A_{8} e^{\beta_{4} x} & \\ -\frac{p}{\alpha} x+\frac{p}{\alpha^{2}}\left(\mu+\lambda \mu_{m}\right), & \text { if } \min \{d, 0\} \leq x \leq 0, \\ \left(A_{3}+A_{7}\right) e^{\beta_{3} x}+\left(A_{4}+A_{8}\right) e^{\beta_{4} x}+A_{5} e^{\beta_{1} x}+A_{6} e^{\beta_{2} x} & \\ +\frac{h}{\alpha} x-\frac{h}{\alpha^{2}}\left(\mu+\lambda \mu_{m}\right), & \text { if } 0 \leq x \leq u, \\ \phi(u)+k_{2}(x-u), & \text { if } u \leq x,\end{cases}
$$

where the constants $A_{i}, i=1, \cdots, 4$, are solutions of (35). Furthermore, the policy parameters $d$, $D, U, u$ and constants $A_{i}, i=5, \cdots, 8$, are given by the solutions of the equations (24), (25) and equations

$$
\left(\begin{array}{cccc}
\frac{e^{\beta_{1} d}}{\eta_{1}+\beta_{1}} & \frac{e^{\beta_{2} d}}{\eta_{1}+\beta_{2}} & \frac{e^{\beta_{3} d}}{\eta_{1}+\beta_{3}} & \frac{e^{\beta_{4} d}}{\eta_{1}+\beta_{4}} \\
e^{\beta_{1} d} & e^{\beta_{2} d} & e^{\beta_{3} d} & e^{\beta_{4} d} \\
e^{\beta_{1} u} & e^{\beta_{2} u} & e^{\beta_{3} u} & e^{\beta_{4} u} \\
\frac{e^{\beta_{1} u}}{\eta_{2}-\beta_{1}} & \frac{e^{\beta_{2} u}}{\eta_{2}-\beta_{2}} & \frac{e^{\beta_{3} u}}{\eta_{2}-\beta_{3}} & \frac{e^{\beta_{4} u}}{\eta_{2}-\beta_{4}}
\end{array}\right)\left(\begin{array}{c}
A_{5} \\
A_{6} \\
A_{7} \\
A_{8}
\end{array}\right)=\left(\begin{array}{c}
-\frac{A_{1}}{\eta_{1}+\beta_{1}} e^{\beta_{1} d}-\frac{A_{2}}{\eta_{2}+\beta_{2}} e^{\beta_{2} d}+\frac{1}{\eta_{1}} \chi_{1}(d, D) \\
-\phi_{1}(d)+\phi(D)+K_{1}+k_{1}(D-d) \\
-\phi_{2}(u)+\phi(U)+K_{2}+k_{2}(u-U) \\
-\frac{A_{3}}{\eta_{2}-\beta_{3}} e^{\beta_{3} u}-\frac{A_{4}}{\eta_{2}-\beta_{4}} e^{\beta_{4} u}+\frac{1}{\eta_{2}} \chi_{2}(u, U)
\end{array}\right)
$$


where

$$
\begin{aligned}
& \chi_{1}(d, D)=\phi(D)+K_{1}+k_{1}\left(D-d+\frac{1}{\eta_{1}}\right)+\frac{p}{\alpha}\left(d-\frac{1}{\eta_{1}}\right)-\frac{p}{\alpha^{2}}\left(\mu+\lambda \mu_{m}\right), \\
& \chi_{2}(u, U)=\phi(U)+K_{2}+k_{2}\left(u-U+\frac{1}{\eta_{2}}\right)-\frac{h}{\alpha}\left(u+\frac{1}{\eta_{2}}\right)+\frac{h}{\alpha^{2}}\left(\mu+\lambda \mu_{m}\right) .
\end{aligned}
$$

Proof: The derivation follows Sepp [15]. The solution to the ordinary differential equation (ODE) in (23) for $d<x<u$ can be represented as

$$
\phi(x)=\phi_{1}(x)+\phi_{2}(x)
$$

where $\phi_{1}(x)$ is a solution of unbounded ODE which is obtained by (32), and $\phi_{2}(x)$ is a solution of homogeneous equation corresponding to ODE with boundary conditions

$$
\left\{\begin{array}{l}
\phi_{2}(d)=\phi(D)+K_{1}+k_{1}(D-d)-\phi_{1}(d) \\
\phi_{2}(u)=\phi(U)+K_{2}+k_{2}(u-U)-\phi_{1}(u) .
\end{array}\right.
$$

For $d \leq x \leq u$, the solution to $\phi_{2}(x)$ has a form

$$
\phi_{2}(x)=\sum_{i=5}^{8} A_{i} e^{\beta_{i-4} x}
$$

Since the function $\phi_{2}(x)$ is continuous at $x=d$ and $x=u$, we have

$$
\left\{\begin{array}{l}
\sum_{i=5}^{8} A_{i} e^{\beta_{i-4} d}=\phi(D)+K_{1}+k_{1}(D-d)-\phi_{1}(d), \\
\sum_{i=5}^{8} A_{i} e^{\beta_{i-4} u}=\phi(U)+K_{2}+k_{2}(u-U)-\phi_{1}(u) .
\end{array}\right.
$$

In addition, the function $\phi_{2}(x)$ satisfies (41). Thus, by substituting (68) into (41), we obtain

$$
\left\{\begin{array}{l}
\sum_{i=5}^{8} \frac{A_{i}}{\eta_{2}-\beta_{i-4}} e^{\beta_{i-4} u}=-\sum_{i=3}^{4} \frac{A_{i}}{\eta_{2}-\beta_{i}} e^{\beta_{i} u}+\frac{1}{\eta_{2}}\left\{\phi(U)+K_{2}+k_{2}\left(u-U+\frac{1}{\eta_{2}}\right)+\chi_{2}\right\}, \\
\sum_{i=5}^{8} \frac{A_{i}}{\eta_{1}+\beta_{i-4}} e^{\beta_{i-4} d}=-\sum_{i=1}^{2} \frac{A_{i}}{\eta_{1}+\beta_{i}} e^{\beta_{i} d}+\frac{1}{\eta_{1}}\left\{\phi(D)+K_{1}+k_{1}\left(D-d+\frac{1}{\eta_{1}}\right)+\chi_{1}\right\},
\end{array}\right.
$$

where $\chi_{1}$ and $\chi_{2}$ are given by (65) and (66), respectively. Hence, the coefficients $A_{i}, i=5, \cdots, 8$ and the thresholds $d, D, U, u$ are determined as the solution to the system (24), (25) and (64).

From (63), the jumps in cash demand have an impact on the value function $\phi(x)$ for $x \in[d, u]$. We consider some limited cases of (63) to understand the effect of the parameters of jumps on the value function. For $\eta_{1} \rightarrow \infty, \beta_{4} \rightarrow-\infty$ and $A_{3}=A_{7}=p_{u}=0$, the first equation in the system (64) vanishes and the function $\phi(x)$ is reduced to the case in the presence of only negative jump of cash demand. For $\eta_{2} \rightarrow \infty, \beta_{1} \rightarrow-\infty$ and $A_{1}=A_{5}=p_{d}=0$, the fourth equation in the system (64) vanishes and the function $\phi(x)$ is reduced to the case in the presence of only positive jump of cash demand. When $\eta_{1} \rightarrow \infty$ and $\eta_{2} \rightarrow \infty$ or $\lambda \rightarrow 0$, the function $\phi(x)$ reduces to the model without jump (Constantinides and Richard [8]).

Theorem 4 Suppose that Assumption 2 holds. Then the function $\phi$ obtained in (63) is a viscosity solution of (19). 
Proof: First, we show that $\phi$ is a viscosity subsolution:

$$
\max \left\{\mathcal{L} \psi\left(x_{0}\right)-C\left(x_{0}\right)-\mathcal{K} \phi\left(x_{0}\right), \phi\left(x_{0}\right)-\mathcal{M} \phi\left(x_{0}\right)\right\} \leq 0 .
$$

Let $\psi \in C^{2}(\mathbb{R}), \psi \leq \phi$ and $\phi\left(x_{0}\right)=\psi\left(x_{0}\right)$. Consider $\phi(x) \leq \mathcal{M} \phi(x)=\inf _{\xi}\{T(\xi)+\phi(x+\xi)\}$ under three cases $x_{0} \leq d, d<x_{0}<u$ and $u \leq x_{0}$. If $x_{0} \leq d$ or $u \leq x_{0}$, we have $\phi(x)=\mathcal{M} \phi(x)$ if and only if $\xi=D-x_{0}$ for $x_{0} \leq d$ or $\xi=U-x_{0}$ for $u \leq x_{0}$. If $d<x_{0}<u$, we obtain $\phi(x)<\mathcal{M} \phi(x)$ with $\xi=0$. Thus, we have $\phi(x) \leq \mathcal{M} \phi(x)$. Next we consider $\mathcal{L} \phi(x)-\mathcal{K} \phi\left(x_{0}\right) \leq C(x)$. If $d<x_{0}<u$, then $\phi-\psi$ is $C^{2}$ at $x=x_{0}$ and has a local maximum at $x_{0}$, thus $\phi^{\prime \prime}\left(x_{0}\right)-\psi^{\prime \prime}\left(x_{0}\right) \leq 0$. Thus, we have

$$
\begin{aligned}
\mathcal{L} \psi\left(x_{0}\right)-C\left(x_{0}\right)-\mathcal{K} \phi\left(x_{0}\right) & =-\frac{1}{2} \sigma^{2} \psi^{\prime \prime}\left(x_{0}\right)+\mu \psi^{\prime}\left(x_{0}\right)+\alpha \psi\left(x_{0}\right)-C\left(x_{0}\right)-\mathcal{K} \phi\left(x_{0}\right) \\
& \leq-\frac{1}{2} \sigma^{2} \phi^{\prime \prime}\left(x_{0}\right)+\mu \phi^{\prime}\left(x_{0}\right)+\alpha \phi\left(x_{0}\right)-C\left(x_{0}\right)-\mathcal{K} \phi\left(x_{0}\right) \\
& =\mathcal{L} \phi\left(x_{0}\right)-C\left(x_{0}\right)-\mathcal{K} \phi\left(x_{0}\right) \\
& =0 .
\end{aligned}
$$

If $x_{0} \leq d$, then we have $\phi\left(x_{0}\right)=\phi(d)+k_{1}\left(d-x_{0}\right), \phi^{\prime}\left(x_{0}\right)=-k_{1}$ and $\phi^{\prime \prime}\left(x_{0}\right)=0$. Thus, we obtain

$$
\begin{aligned}
\mathcal{L} \psi\left(x_{0}\right)-C\left(x_{0}\right)-\mathcal{K} \phi\left(x_{0}\right) & \leq-\frac{1}{2} \sigma^{2} \phi^{\prime \prime}\left(x_{0}\right)+\mu \phi^{\prime}\left(x_{0}\right)+\alpha \phi\left(x_{0}\right)-C\left(x_{0}\right)-\mathcal{K} \phi\left(x_{0}\right) \\
& =-\mu k_{1}+\alpha \phi(d)+\alpha k_{1}\left(d-x_{0}\right)-C\left(x_{0}\right)-\mathcal{K} \phi\left(x_{0}\right) .
\end{aligned}
$$

Similar arguments apply to (59) in the proof of Lemma 9 , for $x_{0} \leq d$, we have $\mathcal{K} \phi\left(x_{0}\right) \geq \mathcal{K} \phi(d)-$ $N\left(d-x_{0}\right)$. For a small $N$, we obtain

$$
\begin{aligned}
\mathcal{L} \psi\left(x_{0}\right)-C\left(x_{0}\right)-\mathcal{K} \phi\left(x_{0}\right) \leq & -\mu k_{1}+\alpha \phi(d)+\alpha k_{1}\left(d-x_{0}\right)-C\left(x_{0}\right)-\mathcal{K} \phi(d) \\
= & \alpha \phi(d)+\mu \phi^{\prime}(d)-\frac{1}{2} \phi^{\prime \prime}(d)+p d-\mathcal{K} \phi(d) \\
& -\mu\left(k_{1}+\phi^{\prime}(d)\right)+\frac{1}{2} \phi^{\prime \prime}(d)+\left(p-\alpha k_{1}\right)\left(x_{0}-d\right) \\
< & 0 .
\end{aligned}
$$

The last inequality follows from $\alpha \phi(d)+\mu \phi^{\prime}(d)-(1 / 2) \phi^{\prime \prime}(d)+p d-\mathcal{K} \phi(d)=0, k_{1}+\phi^{\prime}(d)=0$, $(1 / 2) \phi^{\prime \prime}(d) \leq 0$ and $p-\alpha k_{1}>0$ by Assumption 2 (ii). Similarly, we prove that $\mathcal{L} \psi\left(x_{0}\right)-C\left(x_{0}\right)-$ $\mathcal{K} \phi\left(x_{0}\right)<0$ for $u \leq x_{0}$.

Second, we prove that $\phi$ is a viscosity supersolution:

$$
\max \left\{\mathcal{L} \psi\left(x_{0}\right)-C\left(x_{0}\right)-\mathcal{K} \phi\left(x_{0}\right), \phi\left(x_{0}\right)-\mathcal{M} \phi\left(x_{0}\right)\right\} \geq 0
$$

Let $\psi \in C^{2}(\mathbb{R}), \psi \leq \phi$ and $\psi\left(x_{0}\right)=\phi\left(x_{0}\right)$. Since we always have $\phi\left(x_{0}\right) \geq \mathcal{M} \phi\left(x_{0}\right)$, it suffices to prove that $\mathcal{L} \psi\left(x_{0}\right)-C\left(x_{0}\right)-\mathcal{K} \phi\left(x_{0}\right) \geq 0$. If $d<x_{0}<u$, then $\phi-\psi$ is $C^{2}$ at $x=x_{0}$ and has a local minimum at $x_{0}$. Thus, we have $\phi^{\prime \prime}\left(x_{0}\right)-\psi^{\prime \prime}\left(x_{0}\right) \geq 0$. It gives $\mathcal{L} \psi\left(x_{0}\right)-C\left(x_{0}\right)-\mathcal{K} \phi\left(x_{0}\right) \geq 0$. For $x_{0} \leq d$ and $u \leq x_{0}$, as in the proof of a viscosity subsolution, we can show that $\mathcal{L} \psi\left(x_{0}\right)-C\left(x_{0}\right)-\mathcal{K} \phi\left(x_{0}\right)>0$. Therefore, the function $\phi$ is a viscosity solution.

By Theorems 3 and 4, we can prove the existence and form of optimal policy for the cash management problem (9). 
Table 1: Base parameters.

\begin{tabular}{lclc}
\hline Drift $(\mu)$ & 0.1 & Proportional cost $\left(k=k_{1}=k_{2}\right)$ & 0.2 \\
Volatility $(\sigma)$ & 0.3 & Fixed cost $\left(K=K_{1}=K_{2}\right)$ & 0.8 \\
Discount rate $(\alpha)$ & 0.01 & Intensity $(\lambda)$ & 0.3 \\
Holding cost $(h)$ & 0.1 & Mean of jump size $\left(1 / \eta_{1}=1 / \eta_{2}\right)$ & $1 / 100$ \\
Penalty cost $(p)$ & 0.4 & Probabilities of jump $\left(p_{u}=p_{d}\right)$ & 0.5 \\
\hline
\end{tabular}

\section{Numerical Example}

In this section, we provide some numerical examples to illustrate the effect of the jump risk on the optimal policy. We restrict the cash management model defined in previous section to the one in which the transaction is only allowed to revise the cash level upward. Let $u \rightarrow \infty$ and set $A_{5}=A_{6}=0$ in (63), since $\beta_{1}, \beta_{2}>0$ and the value function $\phi$ is Lipschitz. Thus, the optimal policy can be reduced to the single band policy $(d, D)$. Table 5 reports the parameters used in the computation.

The sensitivity analysis for the thresholds $d$ and $D$ are performed by varying different parameters. Figure 3 shows the effect of the jump parameters on the thresholds. Since the mean of size and probability for positive jump is equivalent to that for negative one, the change of the intensity $\lambda$ does not impact on the thresholds. In case that the probability of positive jump $p_{u}$ is large, it is likely to be short on cash. It leads to increase the thresholds and the cash is kept at a high level. The figures at the bottom of Figure 3 represent the thresholds with respect to the mean of positive and negative jump sizes $1 / \eta_{1}, 1 / \eta_{2}$, respectively. A large size of positive (negative) jump implies that a large amount of outflow (inflow). To prevent the short on cash, the cash level after adjustment $D$ is high as the positive jump size increases. The change of the negative jump size $1 / \eta_{2}$ has an impact on the both of upper and lower control limits $D$ and $d$. The small value of $d$ implies the long interval between the cash adjustments. Thus, as a negative jump size $1 / \eta_{2}$ increases, the cash inventory increases and so the time interval of the adjustment is longer due to reduce the transaction cost. On the other hand, the small value of $D$ means the small size of cash inventory. So, the inventory is decreasing for reducing the holding cost if the negative jump size is large. These different roles of the threshold lead to non-monotonicity of the amount of cash adjustment $D-d$ with $1 / \eta_{2}$. We can see that the difference $D-d$ decreases while the jump size $1 / \eta_{2}$ is relatively small (i.e. 0.05 to 0.03 ), and increases while $1 / \eta_{2}$ is relatively large (i.e. 0.03 to 0.05 ). When $1 / \eta_{2}$ is relatively small, the increase of cash level by the jumps is also small. Thus, it is better to keep large amount of cash inventory to prevent cash shortage. Hence, the difference $D-d$ is decreasing in $1 / \eta_{2}$. However, when $1 / \eta_{2}$ is relatively large, the cash inventory becomes large enough if the jumps occur. Although the possibility of the cash shortage is low, the upper level $D$ is not significantly decreasing because there is a possibility of the positive jumps. In addition, the interval of adjustment becomes long since $d$ is decreasing in $1 / \eta_{2}$. For there reasons, the difference $D-d$ increases as $1 / \eta_{2}$ increases.

\section{Concluding Remarks}

In this paper we considered the cash management model in which the cash level suddenly increase or decrease in a large amount. We formulated such a cash management model as an impulse control 

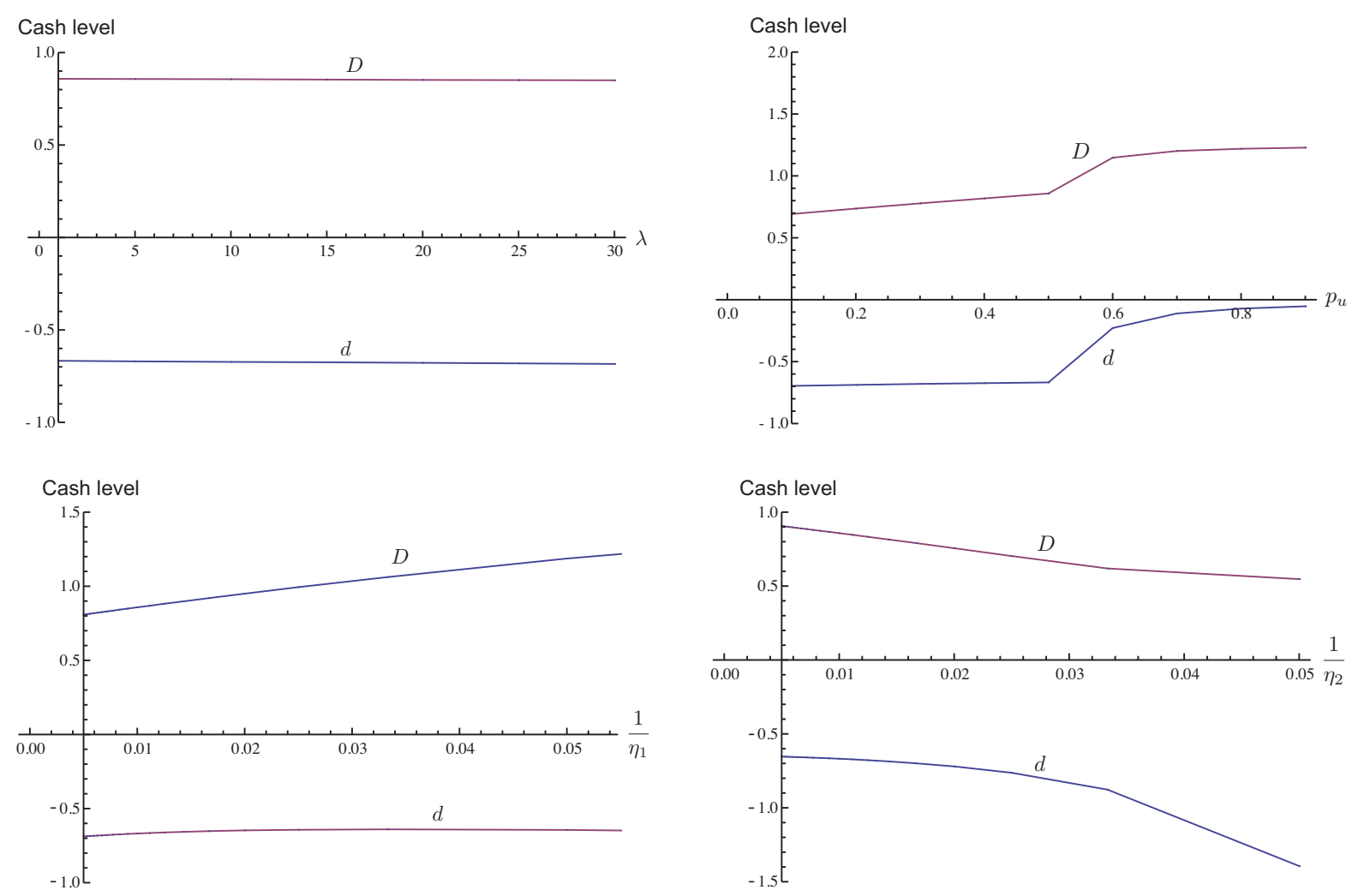

Figure 3: Thresholds $(d, D)$ with respect to the jump parameters.

problem in which the dynamics of the cash demand described by a jump diffusion process. We derived an explicit value function for satisfying the quasi-variational inequality and showed the existence of the optimal policy. In numerical examples, we investigated the effect of the jump on the optimal policy.

There are several topics of further study. One is to show that the existence of an optimal policy for a generalized running cost function and jump diffusion process. Another interesting direction is to study the cash management model with jump diffusion process involving two types of assets: deposits in a bank account and investments in stock. These extensions would be more realistic for applying a cash management model to a practical issue.

\section{A Appendix}

\section{A.1 Derivation of (10)}

Proof: Following Bensoussan (2011, p.288), we express the value function at time $t$ in terms of the value function at the $t+\epsilon$ through dynamic programming. We consider two cases, depending on whether or not $t$ is a stopping time. Then we obtain

$$
\phi(x(t-))=\min \left\{\begin{array}{l}
\inf _{\xi}\left[T(\xi)+E_{x(t)}^{v}[C(x(t))] \epsilon+e^{-\alpha \epsilon} \phi(x(t)+d x)\right], \\
E_{x(t-)}^{v}[C(x(t-))] \epsilon+e^{-\alpha \epsilon} \phi(x(t-)+d x) .
\end{array}\right.
$$

The above equation yields the following inequality:

$$
\phi(x(t-)) \leq E_{x(t-)}^{v}[C(x(t-))] \epsilon+e^{-\alpha \epsilon} \phi(x(t-)+d x) .
$$


Since the cash level is not adjusted for a small amount of time $\epsilon$, we have $d x=x-Z_{\epsilon}$ in the right-hand size of (78). Thus, from $x(t-)=x$ and $e^{-\alpha \epsilon} \approx 1-\alpha \epsilon$, we obtain

$$
\phi(x) \leq \epsilon C(x)+(1-\alpha \epsilon) E\left[\phi\left(x-Z_{\epsilon}\right)\right],
$$

and

$$
E\left[\phi\left(x-Z_{\epsilon}\right)\right]=E\left[\phi\left(x-\mu \epsilon-\sigma W_{\epsilon}-M_{\epsilon}\right)\right] .
$$

By stationarity of the Poisson process, we find that

$$
M_{\epsilon}= \begin{cases}0, & \text { w.p. } 1-\lambda \epsilon, \\ \in(y, y+d y), & \text { w.p. } \lambda \epsilon m(y) d y .\end{cases}
$$

Therefore, we have

$$
E\left[\phi\left(x-Z_{\epsilon}\right)\right] \approx(1-\lambda \epsilon) E\left[\phi\left(x-\mu \epsilon-\sigma W_{\epsilon}\right)\right]+\lambda \epsilon \int_{-\infty}^{\infty} \phi(x-y) m(y) d y .
$$

Let $S(\epsilon)$ be the right hand side of (79). Expanding $S(\epsilon)$ up to the first order in $\epsilon$, we obtain

$$
S(\epsilon)=S(0)+S^{\prime}(0) \epsilon
$$

Here, $S(0)=\phi(x)$ by $Z_{0}=0$, and

$$
\begin{aligned}
S^{\prime}(0) & =\lim _{\epsilon \rightarrow 0}\left\{C(x)-\alpha E\left[\phi\left(x-Z_{\epsilon}\right)\right]+(1-\alpha \epsilon) \frac{\partial}{\partial \epsilon} E\left[\phi\left(x-Z_{\epsilon}\right)\right]\right\} \\
& =C(x)-\alpha \phi(x)+1 \times \lim _{\epsilon \rightarrow 0} \frac{\partial}{\partial \epsilon} E\left[\phi\left(x-Z_{\epsilon}\right)\right] .
\end{aligned}
$$

Here, we have

$$
\begin{aligned}
\lim _{\epsilon \rightarrow 0} \frac{\partial}{\partial \epsilon} E\left[\phi\left(x-Z_{\epsilon}\right)\right]= & \lim _{\epsilon \rightarrow 0}\left\{-\lambda E\left[\phi\left(x-\mu \epsilon-\sigma W_{\epsilon}\right)\right]+(1-\lambda \epsilon) \frac{\partial}{\partial \epsilon} E\left[\phi\left(x-\mu \epsilon-\sigma W_{\epsilon}\right)\right]\right. \\
& \left.+\lambda \int_{-\infty}^{\infty} \phi(x-y) m(y) d y\right\} .
\end{aligned}
$$

In the second term, we have

$$
\begin{aligned}
\frac{\partial}{\partial \epsilon} E\left[\phi\left(x-\mu \epsilon-\sigma W_{\epsilon}\right)\right] & =\frac{\partial}{\partial \epsilon} \int_{-\infty}^{\infty} \phi(x-\mu \epsilon-\sigma s) \frac{1}{\sqrt{2 \pi \epsilon}} e^{-\frac{s^{2}}{2 \epsilon}} d s \\
& =\frac{\partial}{\partial \epsilon} \int_{-\infty}^{\infty} \phi(x-\mu \epsilon-\sigma \sqrt{\epsilon} s) \psi_{0}(s) d s \\
& =-\int_{-\infty}^{\infty}\left(\mu+\frac{1}{2} \epsilon^{-\frac{1}{2}} \sigma s\right) \phi^{\prime}(x-\mu \epsilon-\sigma s \sqrt{\epsilon}) \psi_{0}(s) d s
\end{aligned}
$$

where $\psi_{0}(\cdot)$ is the standard normal distribution. Thus, we have

$$
\begin{aligned}
\lim _{\epsilon \rightarrow 0} \frac{\partial}{\partial \epsilon} E\left[\phi\left(x-\mu \epsilon-\sigma W_{\epsilon}\right)\right] & =-\mu \phi^{\prime}(x)-\frac{1}{2} \sigma \lim _{\epsilon \rightarrow 0} \frac{1}{\sqrt{\epsilon}} \int_{-\infty}^{\infty} s \phi^{\prime}(x-\mu \epsilon-\sigma s \sqrt{\epsilon}) \psi_{0}(s) d s \\
& =-\mu \phi^{\prime}(x)+\frac{1}{2} \sigma^{2} \phi^{\prime \prime}(x) .
\end{aligned}
$$

The last inequality is obtained by applying L'Hopital's rule. So, we obtain

$$
S^{\prime}(0)=C(x)-\phi(x)-\lambda \phi(x)-\mu \phi^{\prime}(x)+\frac{1}{2} \sigma^{2} \phi^{\prime \prime}(x)+\lambda \int_{-\infty}^{\infty} \phi(x-y) m(y) d y .
$$


Hence, by (83), (79) can be rewritten as follows:

$$
\mathcal{L} \phi(x)-\mathcal{K} \phi(x) \leq C(x),
$$

where the operators $\mathcal{L}$ and $\mathcal{K}$ are defined in (12) and (13), respectively.

If we adjust the cash level by the discretionary amount of size $\xi$, then the cash level becomes $x+\xi$ and we write the inequality

$$
\phi(x) \leq T(\xi)+\phi(x+\xi) .
$$

Thus, we have

$$
\phi(x) \leq \mathcal{M} \phi(x),
$$

where $\mathcal{M}$ is given by (11). Since one of the two decisions must be taken, we have (10) as a complementarity slackness condition.

\section{A.2 Confirmation of Assumption 1}

(i) The differential notation for process $Z_{t}$ defined in (1) is given by

$$
d Z_{t}=\mu d t+\sigma d W_{t}+\int_{\mathbb{R}} z(N(d t, d z)-m(d z) d t), \quad Z_{0}=z .
$$

Thus, Lipschitz conditions are given by

$$
\left\{\begin{array}{l}
|\mu(x)-\mu(y)|=|\mu-\mu|=0 \leq I_{\mu}|x-y|, \\
|\sigma(x)-\sigma(y)|=|\sigma-\sigma|=0 \leq I_{\sigma}|x-y|, \\
|j(x, z)-j(y, z)|=|z-z|=0 \leq I_{j}(z)|z-y| .
\end{array}\right.
$$

(ii) Since the running cost is defined as a linear function in (7), we have

$$
\begin{aligned}
& |C(x)-C(y)|=h|x-y| \leq I_{f}|x-y|, \\
& |C(x)-C(y)|=p|x-y| \leq I_{f}|x-y|
\end{aligned}
$$

(iii) By the transaction costs function (6), we obtain

$$
\left\{\begin{array}{l}
\inf _{\xi \in \mathbb{R}} T(\xi)=\min \left\{K_{1}, K_{2}\right\}>0, \\
T \in C(\mathbb{R} \backslash\{0\}), \\
|T(\xi)| \rightarrow \infty, \text { as }|\xi| \rightarrow \infty, \\
T\left(\xi_{1}\right)+T\left(\xi_{2}\right)=T\left(\xi_{1}+\xi_{2}\right)+K_{1}, \text { if } \xi_{1}, \xi_{2} \geq 0, \\
T\left(\xi_{1}\right)+T\left(\xi_{2}\right) \geq T\left(\xi_{1}+\xi_{2}\right)+K_{2}, \text { if } \xi_{1} \geq 0>\xi_{2} .
\end{array}\right.
$$

(iv) From (91), we can take constants $I_{\mu}, I_{\sigma}$ and $I_{j}$ satisfying the condition (iv).

\section{Acknowledgment}

We are very grateful to an anonymous referee for his or her insightful and detailed comments that substantially improved the paper. This paper is supported in part by a JSPS Grant-in-Aid for Young Scientists (B) (26870643). 


\section{References}

[1] Baccarin, S. (2002): Optimal Impulse Control for Cash Management with Quadratic Holdingpenalty Costs. Decisions in Economics and Finance, 25, 19-32.

[2] Baccarin, S. (2009): Optimal Impulse Control for a Multidimensional Cash Management System with Generalized Cost Functions. European Journal of Operational Research, 196, 198-206.

[3] Bar-Ilan, A., Perry, D. and Stadje, W. (2004): A Generalized Impulse Control Model of Cash Management. Journal of Economic Dynamics \&3 Control, 28, 1013-1033.

[4] Baumol, W. (1952): The Transactions Demand for Cash: An Inventory-theoretic Approach. Quarterly Journal of Economics, 66, 545-556.

[5] Benkherouf, L. and Bensoussan, A. (2009): Optimality of an $(s, S)$ Policy with Compound Poisson and Diffusion Demands: A Quasi-variational Inequalities Approach. SIAM Journal on Control and Optimization, 48, 756-762.

[6] Bensoussan, A. (2011): Dynamic Programming and Inventory Control. IOS Press.

[7] Bensoussan, A., Liu, R. H. and Sethi, S. P. (2006): Optimality of an $(s, S)$ Policy with Compound Poisson and Diffusion Demand: A QVI Approach. SIAM Journal on Control and Optimization, 44, 1650-1676.

[8] Constantinides, G. and Richards, S. (1978): Existence of Optimal Simple Policies for Discounted Cost Inventory and Cash Management in Continuous Time. Operations Research, 26, 620-636.

[9] Davis, M., Gin, X. and Wu, G. (2010): Impulse Control of Multidimensional Jump Diffusions. SIAM Journal on Control and Optimization, 48, 5276-5293.

[10] Guo, X. and Wu, G. (2009): Smooth Fit Principle for Impulse Control of Multidimensional Diffusion Processes. SIAM Journal on Control and Optimization, 48, 594-617.

[11] Korn, R. (1997): Optimal Impulse Control When Control Actions Have Random Consequences. Mathematics of Operations Research, 22, 639-667.

[12] Kou, S. G. and Wang, H. (2003): Option Pricing Under a Double Exponential Jump Diffusion Model. Management Science, 50, 1178-1192.

[13] Miller, M. and Orr, D. (1966): A Model of the Demand for Money by Firms. The Quarterly Journal of Economics, 81, 413-435.

[14] Øksendal, B. and Sulem, A. (2007): Applied Stochastic Control of Jump Diffusions. Springer, Berlin and Heidelberg.

[15] Sepp, A. (2004): Analytical Pricing of Double-barrier Options Under a Double-exponential Jump Diffusion Process: Applications of Laplace Transform. International Journal of Theoretical and Applied Finance, 7, 151-175. 